\title{
La gobernanza del agua como marco integrador para el cumplimiento de los Objetivos de Desarrollo Sostenible en Latinoamérica
}

\section{Water governance as an integrating framework for the fulfillment of the sustainable development goals clean in Latin America}

\author{
Gabriel Fernández-Vargas ${ }^{1}$
}

11Ing. Ambiental, M.Sc., Ph.D. (c). Universidad del Valle. Santiago de Cali - Valle del Cauca, Colombia; e-mail gabriel.fernandez@correounivalle.edu.co; (Dhttps://orcid.org/0000-0003-4761-7166

Cómo citar: Fernández-Vargas, G. 2020. La gobernanza del agua como marco integrador para el cumplimiento de los Objetivos de Desarrollo Sostenible en Latinoamérica Rev. U.D.C.A Act. \& Div. Cient. 23(2):e1561. http://doi.org/10.31910/rudca.v23.n2.2020.1561

Artículo de acceso abierto publicado por Revista U.D.C.A Actualidad \& Divulgación Científica, bajo una licencia Creative Commons CC BY-NC 4.0

Publicación oficial de la Universidad de Ciencias Aplicadas y Ambientales U.D.C.A, Institución de Educación Superior Acreditada de Alta Calidad por el Ministerio de Educación Nacional.

Recibido: Mayo 23 de 2020 Aceptado: Octubre 16 de 2020 Editado por: Adriana Posada Arrubla

\section{RESUMEN}

El cumplimiento del Objetivo de Desarrollo Sostenible, ODS, No 6 "Agua Limpia y Saneamiento", promulgado en 2015 por la ONU, requiere de nuevos enfoques y marcos conceptuales, que articulen e integren, de manera práctica, las intervenciones en el sector de Agua Potable, Saneamiento e Higiene (APSH), con la Gestión Integrada de Recursos Hídricos (GIRH), según los contextos específicos de cada región. En este sentido, la gobernanza del agua proporciona un marco conceptual, que permite analizar y potenciar la integración entre los sectores de APSH con la GIRH, en el marco del cumplimiento de los ODS. Para ello, se documentaron varios casos de estudio en Latinoamérica, donde se observó la integración sectorial, a partir del modelo de funciones de Gobernanza del Agua, desarrollado por Water Governance Facility - WGF. Si bien cada uno de los casos de estudio se enmarcan en alguna función específica de la gobernanza del agua, todos requieren, al menos, de otra función, para poder lograr su finalidad, siendo la "Coordinación", la más relevante, observada para el logro de los resultados obtenidos.
Palabras clave: Objetivos de Desarrollo Sostenible; Gobernanza del agua; Agua potable y saneamiento.

\section{ABSTRACT}

The fulfillment of the Sustainable Development Goal, SDG, No 6 "Clean Water and Sanitation", promulgated in 2015 by the UN, requires new approaches and conceptual frameworks that articulate and integrate in a practical way the interventions of Drinking Water, Sanitation and Hygiene - WASH, with the Integrated Water Resources Management, IWRM, according to the specific contexts of each territory. In this sense, water governance provides a conceptual framework that allows analyzing and enhancing integration between WASH sectors with IWRM, within the framework of the fulfillment of the SDGs. For this, several case studies in Latin America were documented where sectoral integration was observed, based on the Water Governance Facility model of Water Governance. Although each of the case studies is framed in some specific function of 
water governance, all require at least one other function in order to achieve their purpose, with "Coordination" being the most relevant observed for the achievement of obtained results.

Keywords: Sustainable Development Goals; Water governance; Integrated water resources management.

\section{INTRODUCCIÓN}

En el 2015, la Asamblea General de las Naciones Unidas aprobó la Agenda de Desarrollo Sostenible 2030, compuesta por 17 objetivos y 169 metas, que sustituyen los Objetivos de Desarrollo del Milenio. Para el caso del ODS 6 "Garantizar la disponibilidad y la gestión sostenible del agua y el saneamiento para todos", se establecieron ocho metas asociadas, las cuales, se pueden agrupar en dos temas/ sectores principales: i) Agua Potable, Saneamiento e Higiene (APSH) y ii) Gestión Integrada de Recursos Hídricos (GIRH), así como también los medios de implementación, que se consideran transversales a los dos sectores anteriores, como se observa en la figura 1.

La manera como está planteado el ODS 6 reconoce que los desafíos asociados al agua y saneamiento se pueden cumplir, mediante la adopción de enfoques más integrados para la gestión y asignación de los recursos hídricos, incluida la protección de los ecosistemas, sobre los que dependen las sociedades y las economías (Valencia et al. 2019); no obstante, si bien a primera vista los temas de APSH y GIRH parecen estar "naturalmente" interconectados, dado que dependen del mismo recurso natural, la realidad muestra otra cosa. Ello es más evidente en el nivel ministerial en países latinoamericanos, donde cada tema se concibe como un "sector hídrico" diferente, con sus propias políticas, planes de acción y presupuestos. Incluso, desde el punto de vista conceptual, se aprecian diferencias entre estos sectores (UNICEF et al. 2016; GWP, 2019). Esta división sectorial histórica al interior de la gestión del agua, entre otros factores, ha propiciado que la crisis actual del agua se reconozca cada vez más como una crisis de gobernanza del agua (UNESCO, 2006).

En tal sentido, el cumplimiento del ODS 6 requiere de enfoques más integrados para abordar la sostenibilidad en la prestación de servicios de APSH, en un mundo con una inseguridad hídrica, cada vez mayor (Wetlands International, 2017). Es por eso, que forjar mejores vínculos y lograr una verdadera integración entre el sector de APSH y la GIRH es un desafío, pero, a su vez, la mejor oportunidad para alcanzar el cumplimiento del ODS 6 al 2030, a nivel global. Para ello, el marco de las funciones de gobernanza del agua brinda un marco de análisis, que permite el establecimiento de puentes o vínculos entre APSH y la GIRH, con el fin de potenciar el cumplimiento del ODS 6, a nivel global, en un futuro cercano.

En tal sentido, este artículo tiene como objetivo identificar y analizar la vinculación entre los sectores de APSH y GIRH en contexto latinoamericano, en el marco del ODS6, mediante el modelo de gobernanza del agua, propuesto por SIWI (2018).

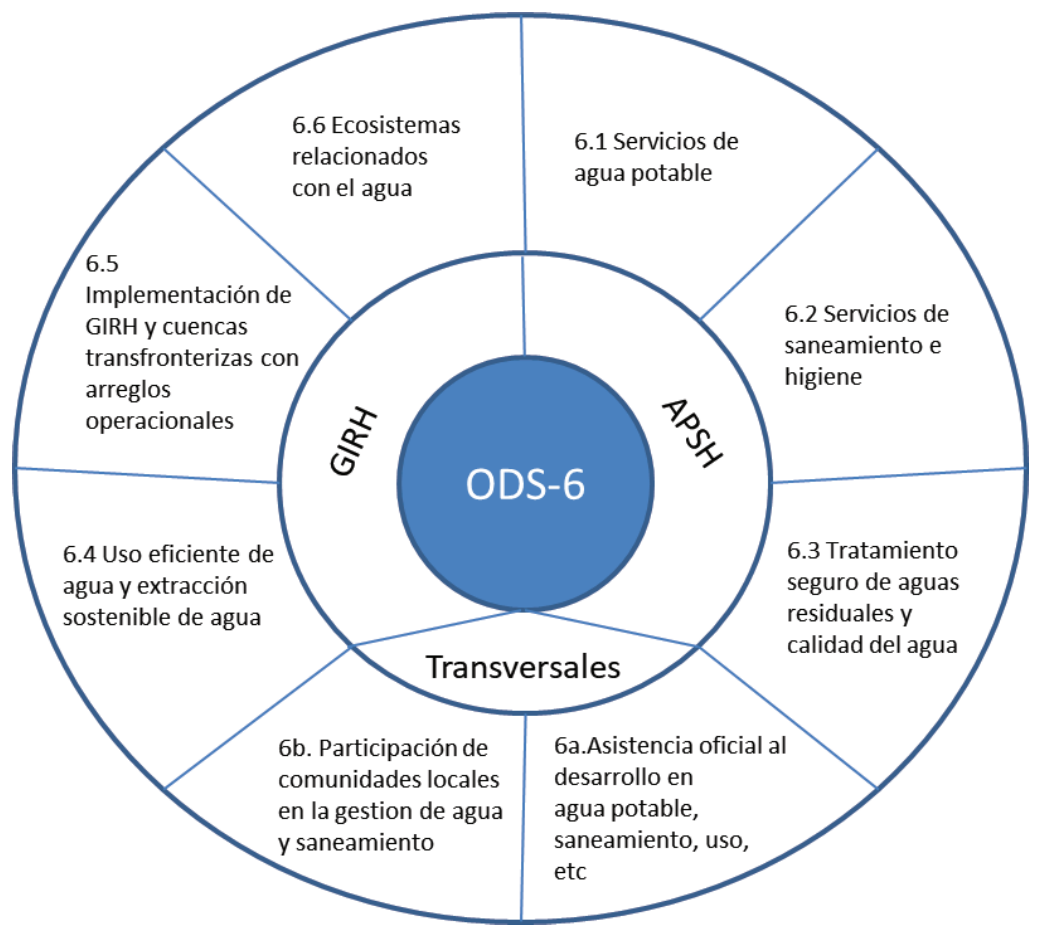

Figura 1. Clasificación de metas del ODS 6 por sectores. 


\section{MATERIALES Y METODOS}

El área de estudio de esta investigación, se concentra en la región andina de Suramérica, en la cual, se ubican Venezuela, Colombia, Ecuador, Perú, Bolivia, Chile y Argentina, donde una adecuada prestación de servicios de agua y saneamiento, para todos sus habitantes y una gestión integrada de recursos hídricos, son cada vez más complejos de alcanzar.

La metodología utilizada partió de la revisión de literatura sobre APSH, GIRH y los principales modelos de la gobernanza del agua, desarrollados a nivel global (OCDE, 2012; Havekes et al. 2013; Flammini et al. 2014; SIWI, 2018; GWP, 2019; SWA, 2020). A partir de ello, se seleccionó el modelo diseñado por SIWI (2018), que define un conjunto de funciones que aplican, tanto para el sector de APSH como de la GIRH, lo que permite identificar y analizar, de mejor manera, los vínculos entre estos sectores hídricos.

Luego, se seleccionaron casos potenciales a documentar sobre la vinculación de los sectores APSH y GIRH en Latinoamérica, a partir de lo cual, se realizó una revisión de literatura específica y se desarrollaron cuatro entrevistas virtuales con los responsables de algunos casos identificados, con quienes se ampliaron y precisaron aspectos técnicos de cada caso, que se detallan más adelante. Finalmente, la información recolectada se organizó, se sistematizó y se analizó, con base en el marco de Gobernanza del Agua propuesto, por SIWI (2018).

Para el desarrollo del artículo, se tuvieron en cuenta las siguientes definiciones y conceptos relacionados con la investigación:

Agua potable, saneamiento e higiene. Se puede definir el sector de APSH, como el conjunto de actividades interdependientes, que incluyen la provisión de agua para consumo humano y doméstico, la disposición de residuos líquidos y domésticos de origen humano y las prácticas de higiene asociadas. Según UNICEF (2016), las funciones clave de gobernanza de este sector se pueden agrupar en las siguientes: i) Política sectorial y estrategia; ii) Arreglos institucionales; 3) Planificación, seguimiento y revisión; iv) Presupuesto y financiamiento; y iv) capacidad del sector desarrollo.

Gestión integrada de recursos hídricos. Hoy, se sabe con algún grado de certeza, que la gestión del agua es muy compleja y que, idealmente, debe ser manejada de manera integral, teniendo en cuenta la eficiencia, la equidad y la protección del ambiente (Giordano \& Shah, 2014). Por ello, para abordar esta crisis, se requieren nuevas miradas y enfoques intersectoriales para la gestión del agua como, por ejemplo, la gestión integrada de los recursos hídricos (GIRH) (Restrepo, 2004). Este concepto, que busca ser atractivo y lógico, se basa en que los usos del agua son múltiples e interdependientes y que deben ser considerados en conjunto (CapNet et al. 2004). Así, esta GIRH, se concibe como "un proceso que promueve el manejo y el desarrollo coordinado del agua, la tierra y los recursos relacionados, con el fin de maximizar el bienestar social y económico" (GWP, 2000); no obstante, es necesario aceptar que este concepto no se adapta a todas las situaciones o condiciones de la gestión del agua, por lo que hay que reconocerlo como una herramienta e incluir otras alternativas en el discurso y en la acción para afrontar la "crisis global del agua" (Giordano \& Shah, 2014).

La GIRH significa, entonces, la reconciliación de las necesidades humanas básicas, asegurando acceso y equidad, con desarrollo económico y la imperativa integridad ecológica, mientras respeta compromisos transfronterizos (Zaag, 2005). Para ello, forjar mejores vínculos y lograr una verdadera integración entre los sectores de Agua Potable, Saneamiento e Higiene y la GIRH es otra forma de fortalecer la GIRH en su conjunto, incluyendo la participación de base (Butterworth et al. 2010). Así, la inclusión del ODS6 coloca la responsabilidad de la gestión del agua en las manos del sector hídrico y de los actores que utilizan el agua (Shah, 2016).

El marco de la gobernanza del agua. Si bien existen en la actualidad múltiples definiciones y conceptos sobre gobernanza del agua, la definición más utilizada, a nivel mundial, es la proporcionada por la Asociación Mundial del Agua, que la plantea como "el conjunto de sistemas políticos, sociales, económicos y administrativos establecidos para desarrollar y gestionar los recursos hídricos y la prestación de servicios de agua en diferentes estratos de la sociedad" (Rogers \& Hall, 2003). Esta gobernanza del agua, se constituye a partir de una serie de funciones centrales, que se refieren a una gama de actividades estándar que deben realizar las instituciones del sector (UNDP et al. 2013), para una adecuada gestión del agua.

En el caso del modelo de gobernanza del agua propuesto por el Instituto SIWI (2018), se incluyen, como funciones, las siguientes: i) Política y Estrategia, ii) Coordinación, iii) Preparación y planificación, iv) Financiación, v) Arreglos institucionales, vi) Regulación, vii) Desarrollo de capacidades, viii) Monitoreo, evaluación y aprendizaje, y ix) Gestión de desastres - respuesta y recuperación.

\section{RESULTADOS Y DISCUSION}

La gobernanza del agua como marco integrador de los sectores de Agua potable, Saneamiento e Higiene y la Gestión Integrada de Recursos Hídricos en América Latina. A partir del marco de las funciones clave de la gobernanza del agua propuesto por SIWI (2018), a continuación, se identifican y analizan vínculos entre el sector de APSH y la GIRH en América Latina, a partir de ocho casos de estudio observados en siete países andinos, en cada uno de los cuales, aplica un número de funciones específicas (Tabla 1).

Fondo de Protección del Agua de Quito (Ecuador). El Fondo de Protección del Agua de Quito, FONAG, se originó en el 2000, a través de esfuerzos de The Nature Conservancy, TNC y Fundación Antisana, junto con socios clave, como la Alcaldía de Quito y la Empresa de Agua de Quito (Epmaps) (Goldman-Benner et al. 2012). Si bien el FONAG se creó inicialmente como un mecanismo de financiación de largo término de la conservación de la biodiversidad en la Reserva de la Biosfera Condor (Joslina \& Jepson, 2018), hoy, funciona como un Fondo de Agua, siendo el primero (pionero) de 
Tabla 1. Resumen de los tipos de funciones de gobernanza del agua identificados en los casos de estudio documentados.

\begin{tabular}{|c|c|c|c|c|c|c|c|c|c|}
\hline & $\begin{array}{c}\text { Política y } \\
\text { Estrategia }\end{array}$ & $\begin{array}{l}\text { Coordi- } \\
\text { nación }\end{array}$ & $\begin{array}{c}\text { Preparación } \\
\text { y } \\
\text { planifi- } \\
\text { cación }\end{array}$ & $\begin{array}{l}\text { Finan- } \\
\text { ciación }\end{array}$ & $\begin{array}{l}\text { Arreglos in- } \\
\text { stitucionales }\end{array}$ & Regulación & $\begin{array}{c}\text { Desarrollo } \\
\text { de capaci- } \\
\text { dades }\end{array}$ & $\begin{array}{c}\text { Monitoreo, } \\
\text { Evaluación } \\
\text { y Aprendi- } \\
\text { zaje }\end{array}$ & $\begin{array}{l}\text { Gestión } \\
\text { de de- } \\
\text { sastres } \\
\text { (riesgo) }\end{array}$ \\
\hline $\begin{array}{l}\text { FONAG } \\
\text { Ecuador }\end{array}$ & $\mathrm{x}$ & $\mathrm{x}$ & $\mathrm{x}$ & $\mathbf{X}$ & $\mathrm{x}$ & $\mathrm{x}$ & $\mathrm{x}$ & & \\
\hline $\begin{array}{l}\text { Codeparh } \\
\text { Colombia }\end{array}$ & $\mathrm{x}$ & $\mathbf{x}$ & $\mathrm{x}$ & & $\mathrm{x}$ & & $\mathrm{x}$ & $\mathrm{x}$ & \\
\hline $\begin{array}{l}\text { PNC Bo- } \\
\text { livia }\end{array}$ & $\mathrm{x}$ & $\mathrm{x}$ & $\mathbf{X}$ & $\mathrm{x}$ & & $\mathrm{x}$ & & & \\
\hline $\begin{array}{l}\text { MRSE } \\
\text { Perú }\end{array}$ & $\mathrm{X}$ & $\mathrm{x}$ & & & & $\mathrm{x}$ & & & \\
\hline $\begin{array}{l}\text { Reúso } \\
\text { de aguas } \\
\text { residuales } \\
\text { domésticas } \\
\text { tratadas en } \\
\text { Argentina }\end{array}$ & $\mathrm{x}$ & $\mathrm{x}$ & & & $\mathrm{X}$ & $\mathrm{x}$ & & & \\
\hline $\begin{array}{l}\text { LA-Wet } \\
\text { Net }\end{array}$ & & $\mathrm{x}$ & & $\mathrm{x}$ & & & $\mathbf{x}$ & & \\
\hline $\begin{array}{l}\text { Agenda } \\
\text { Venezuela } \\
2030\end{array}$ & & $\mathrm{x}$ & $\mathrm{x}$ & & $\mathrm{x}$ & & & $\mathbf{x}$ & \\
\hline $\begin{array}{l}\text { Seguridad } \\
\text { hídrica } \\
\text { Chile }\end{array}$ & $\mathrm{x}$ & $\mathrm{x}$ & $\mathrm{x}$ & & $\mathrm{x}$ & & & & $\mathbf{x}$ \\
\hline
\end{tabular}

X: Función principal

x: Función complementaria

su tipo en Latinoamérica y también modelo para la implementación de otros Fondos en la región (Chafla \& Ceron, 2016).

El FONAG permite a los usuarios del recurso hídrico, ubicados aguas abajo (compradores de servicios ecosistémicos), financiar actividades aguas arriba, con el fin de obtener un suministro limpio y regular de agua (Goldman-Benner et al. 2012). En todos los casos, los servicios se originan dentro de ecosistemas naturales, pero son la ordenación y el uso de la tierra por parte de las comunidades humanas en sus territorios específicos, las que determinan la prestación de servicios, siendo estas comunidades los principales proveedores de estos servicios (Goldman-Benner et al. 2012).

Para el funcionamiento del Fondo es vital la participación del sector APSH, a través de la Epmaps (principal aportante), dado que, por normatividad, se dispuso un aporte inicial de USD20.000 para la creación del fideicomiso y, al menos, el 2\% de las tarifas que cobra la empresa de agua, se destina al fondo de agua (Goldman-Benner $e t$ al. 2012). Para ello, fue fundamental la expedición de la Ordenanza Metropolitana 199 de 2006 (actualmente Ordenanza 213 de 2007), la cual, blinda con recursos económicos el funcionamiento de FONAG.
Si bien dentro del esquema de Gobernanza del Agua, el FONAG se relaciona directamente con la función de "Financiación", para el cumplimiento de sus objetivos ha sido clave también la "Coordinación" entre entidades y la "Regulación" normativa. De igual manera, hoy el FONAG también presta asesoramiento técnico y genera información hidroclimática de las cuencas hidrográficas de su área de influencia, con lo cual, se relaciona directamente con otras funciones de gobernanza, como "Desarrollo de capacidades" y "Monitoreo, evaluación y aprendizaje".

Consejo Departamental de Política Ambiental y de Gestión Integral del Recurso Hídrico - CODEPARH Valle del Cauca, Colombia. Se creó mediante la Ordenanza 421 de 2016, como un mecanismo de articulación, asesoría política y gobernanza, con el propósito de garantizar el logro de la visión del desarrollo sostenible a mediano y largo plazo. Este Consejo quedó integrado por representantes de Autoridades Departamentales y Locales, la Autoridad Ambiental Regional, Empresas prestadoras de servicios de agua potable y saneamiento y universidades, entre otros (Gobernación del Valle del Cauca, 2019). 
Como principales resultados de este Consejo, se destaca la formulación de la Política Pública Departamental en Ambiente y Gestión Integral del Recurso Hídrico, adoptada mediante Ordenanza 445 de 2017 y luego modificada por la Ordenanza 446 de 2017 (Gobernación del Valle del Cauca, 2019). Además, se suscribió, en 2017, un Memorando de Intención, firmado por 21 instituciones públicas, privadas y de la sociedad civil, que avaló un plan de Inversión inicial orientado a la restauración y protección de cinco cuencas prioritarias del departamento.

Un aspecto importante para destacar es la participación del sector de APSH en el Codeparh, siendo el caso de la Sociedad de Acueducto y Alcantarillado del Valle del Cauca, Acuavalle ESP, Vallecaucana de Aguas ESP y la Empresa de servicios Públicos de Yumbo (ESPY). Es así como, además de la participación en las mesas locales de coordinación, estas empresas han invertido en obras de captación, conducción, tratamiento y desinfección de agua para consumo humano y en obras de saneamiento básico y construcción de alcantarillados y plantas de tratamiento de aguas residuales (CODEPARH, 2019).

Aun cuando el Codeparh se enmarca, principalmente, dentro de una función de Coordinación en el marco de Gobernanza del Agua (SIWI, 2018), se observa que para su avance se requirió del desarrollo de otras funciones, como Política y Estrategia, Preparación y Planificación, Arreglos Institucionales, entre otras, sin las cuales, no se tendrían los resultados positivos, que hoy presenta este consejo.

Plan Nacional de Cuencas - Bolivia. Teniendo como antecedente la "Guerra del Agua" desatada en Cochabamba, Bolivia, a inicio de la década del 2000 y luego de la expedición de legislación nacional sobre el tema, en 2003, se firma un acuerdo entre los países donantes Holanda, Alemania y Suiza con el gobierno boliviano, para la implementación del Proyecto de Promoción del Programa Nacional de Cuencas (PPPNC), precursor directo del Plan Nacional de Cuencas (PNC) (CTB Enabel, 2019). Posteriormente, con la llegada de Evo Morales a la Presidencia de la República, se crea, en el 2006, el Ministerio del Agua, como respuesta a las demandas de movimientos sociales y los sucesos de la "Guerra por el agua", entidad que consideró tres nuevos subsectores, los cuales, eran riego, agua potable y cuencas (Ruiz \& Gentes, 2008). Con la creación de este Ministerio del Agua, se decide elaborar la primera versión del PNC con la cooperación holandesa y sueca, teniendo como resultado un documento conceptual y estratégico, que enmarca las políticas del Gobierno Nacional en el tema, incluyendo, la participación social en todos los procesos y un enfoque transversal cultural (Danida, 2007).

La versión inicial del PNC reconoce, en 2006, como un problema central de la gestión institucional del agua, la falta de una gestión integrada e intersectorial del recurso hídrico y una visión a largo plazo, en parte, por falta de una estrategia que ubique y vincule al agua potable y saneamiento con el riego y otros sectores (medio ambiente, minería, electricidad, hidrocarburos y forestal) (Ministerio del Agua, 2007). Luego, con una segunda versión, formulada durante el 2013, el PNC se consolida como política del Estado
Plurinacional de Bolivia (CTB Enabel, 2019), que permite adoptar, mediante Resolución Ministerial 0540 del 13 de diciembre de 2013, el Programa Plurianual de Gestión Integrada de Recursos Hídricos, GRH y Manejo Integral de Cuencas, MIC 2013-2017 (Ministerio de Medio Ambiente y Agua, 2013). Finalmente, mediante Resolución Ministerial 0470 del 08 de septiembre de 2017, el Programa Plurianual de GIRH-MIC se ajustó, extendiendo su vigencia del 2017 al 2020 (Ministerio de Medio Ambiente y Agua, 2017).

El PNC de Bolivia es un ejemplo de la función de gobernanza del agua asociada a Preparación y Planeación, en la cual, convergen diferentes actores sociales del país, según sus competencias del orden local, regional y nacional, pero que requiere de otras funciones para su desarrollo, como es el caso de la Coordinación, Política y Estrategia y Financiación, entre otros.

Mecanismos de retribución por servicios ecosistémicos Perú. Con la expedición de la Ley Orgánica para el aprovechamiento sostenible de los recursos naturales (Ley 26821 de 1997), se institucionaliza el tema de los servicios ecosistémicos en el Perú, al incorporar como una obligación del Estado, realizar el inventario y valorización de los recursos naturales y de los servicios ambientales. Luego, la Ley sobre la conservación y el aprovechamiento sostenible de la diversidad biológica (Ley 26839 de 1997), incorpora como una obligación del Estado, la adopción de medidas como mecanismos financieros e instrumentos económicos, para una adecuada gestión de la diversidad biológica en el país; no obstante, es con la expedición de la Ley de Modernización de los Servicios de Saneamiento del Perú (Ley 30045 de 2013), que se da un paso significativo hacia adelante, al definir que las entidades prestadoras de servicios de saneamiento, EPS y la Superintendencia Nacional de Servicios de Saneamiento, SUNASS, debían incluir, en su Plan Maestro Optimizado, mecanismos de compensación ambiental y manejo de cuencas (Congreso de la República del Perú, 2013).

Posteriormente, el marco normativo nacional se ve fortalecido con la expedición de la Ley de Mecanismos de Retribución por Servicios Ecosistémicos, MRSE (Ley 30215 de 2014) y el Decreto Legislativo 1240 de 2015, el cual, modificó la Ley 30045 de 2013. Este Decreto incluyó que la SUNASS, en coordinación con las EPS, debía incorporar en la tarifa MRSE, destinados a asegurar la permanencia de los beneficios generados por los ecosistemas que proveen de agua, para la prestación de los servicios de saneamiento.

Es así como la mayor cantidad de inversión comprometida en MRSE en el país proviene de las EPS, que está directamente relacionado con la promulgación de Ley 30045 y normas complementarias (Quintero \& Pareja, 2015), siendo, en la actualidad, siete EPS, las que tienen incorporado en su tarifa MRSE (Argote, 2019).

Los MRSE de Perú, se constituyen en un caso de aplicación de la función de gobernanza del agua asociada a Política y Estrategia, especialmente, en el desarrollo normativo del orden nacional, pero que también requiere de otras funciones para su desarrollo, como es el caso de la "Coordinación" entre actores sociales relacionados con los MRSE, así como también "Regulación" y "Financiación”, entre otros. 
Reúso de aguas residuales domésticas tratadas en Mendoza Argentina. Una de las más importantes áreas de riego en Argentina, se localiza en la región central de la provincia de Mendoza, con 350.000ha, aproximadamente (Abril et al. 2014). En esta provincia, el reúso de aguas residuales tratadas en agricultura, se realiza desde hace más de cinco décadas, propiciado por la escasez del recurso hídrico, dada las condiciones de baja precipitación y aridez del territorio y la alta demanda de agua para riego, que representa el 80\% del total de la demanda hídrica (Foresi, 2017).

Al respecto, hay que mencionar que la Ley Provincial 6044 de 1993 permitió la prestación de servicios de saneamiento cloacal, compuesto por aguas residuales de origen doméstico, por parte del sector privado y creó un Ente Provincial del Agua y del Saneamiento, EPAS, que le asignó normas de preservación hídrica, junto con el Departamento General de Irrigación (DGI) y las Municipalidades (Legislatura de Mendoza, 2019). Esta ley plantea que las aguas residuales generadas por los tratamientos de saneamiento cloacal, se encuentran bajo la jurisdicción y control del DGI (Pinto, 2012).

Luego, a partir del 2000, la provincia de Mendoza introduce la posibilidad de hacer reúso controlado de aguas residuales tratadas para riego, con la expedición de la Resolución DGI 627 de 2000, que se materializa en 2003, con la creación de las Áreas de Cultivos Restringidos Especiales, ACRE (DGI, 2019b). Un ACRE es un área agrícola destinada al aprovechamiento productivo de las aguas residuales tratadas que provienen de plantas depuradoras de líquidos cloacales, en la cual, está prohibido que el agua salga del área establecida o se libere al uso irrestricto (DGI, 2019a).

Como parte de los arreglos institucionales para el reúso de aguas residuales, el costo de la depuración de los efluentes cloacales, en ambas plantas, lo pagan los usuarios del agua potable, es decir, los ciudadanos de Mendoza a AySAM. Esta empresa, le entrega el agua tratada en forma gratuita nuevamente al DGI, quien la entrega a los usuarios del ACRE respectivo, quienes deben pagar un canon de riego (Foresi, 2017). Otro arreglo institucional es que la Resolución DGI 400 de 2003 define los cultivos permitidos en las ACRE, de acuerdo con el nivel de tratamiento alcanzado para el efluente cloacal y prohíbe, expresamente, el riego con aguas residuales de cultivos de consumo en fresco que se cosechan y distribuyen de forma inmediata (DGI, 2019b).

Si bien la gestión del reúso de aguas residuales tratadas en Mendoza se puede enmarcar, principalmente, en la función de "Arreglos Institucionales" del esquema de Gobernanza del Agua, se observan otras funciones claves para su desarrollo. Es el caso de "Política y estrategia", "Coordinación" y "Regulación", entre otras.

Agenda Venezuela 2030. Según Sinergia (Red Venezolana de organizaciones de la sociedad civil), Venezuela está en situación de Emergencia Humanitaria Compleja desde 2015, expresada en el desmoronamiento de la economía formal y de las estructuras estatales, los conflictos civiles, los riesgos sanitarios y el éxodo en masa de la población (SINERGIA, 2019). Es así, como la Oficina del Alto Comisionado de las Naciones Unidas para los Derechos
Humanos- ACNUDH considera que el desvío de recursos, la corrupción y la falta de mantenimiento en la infraestructura pública, así como la subinversión, han deteriorado servicios básicos, como el transporte público y el acceso a electricidad, agua y gas natural, dando como resultado, violaciones al derecho, a un nivel adecuado de vida, en este país (ACNUDH, 2019).

La crisis asociada al ODS 6 en Venezuela, no se expresa solamente en términos de la prestación de servicios públicos, también es el caso de la gestión de los recursos hídricos, pues este país cuenta con un patrimonio hídrico importante, pero con desequilibrios territoriales y amenazas importantes (Grupo Orinoco, 2018). Además, grandes usuarios, como la agricultura, a escala nacional, tienen dificultades con el suministro de agua, dado que, una gran parte de las tierras agrícolas, no cuentan con sistemas de riego, lo que las convierte en altamente vulnerables a la variabilidad climática (SINERGIA, 2019).

Ante este panorama y la falta de cifras oficiales y las restricciones al acceso de información pública confiable y oportuna (SINERGIA, 2019), una serie de organizaciones de la sociedad civil crearon la Agenda Venezuela 2030, la cual, tiene como misión informar y promover el seguimiento y monitoreo de los ODS en Venezuela, entre ellos el 6 (SINERGIA, 2020). Esta iniciativa ciudadana, se concibe en una plataforma que permite a sus asociados trabajar en equipo, compartir información y visibilizar la situación de los ODS en Venezuela, sin restricciones ni injerencia del gobierno Nacional (Gil-Beroes, 2020).

Según lo anterior, Agenda Venezuela 2030, se constituye en un caso de Monitoreo, Evaluación y Aprendizaje de la función del marco de gobernanza del agua, pero que requiere también de la Coordinación, en este caso, desde las organizaciones de la sociedad civil, con el fin de avanzar en el cumplimiento del ODS 6, en un país en situación de Emergencia Humanitaria Compleja.

Seguridad hídrica en Chile. Si bien Chile se ubica parcialmente con poca o ninguna escasez de agua (Arroyo et al. 2015), cada vez es más recurrente observar en la literatura académica y técnica que este país sufre de escasez de agua (Oppliger et al. 2019; Escenarios hídricos 2030, 2019a).

Frente a este panorama, nace en el 2016, la iniciativa "Escenarios hídricos 2030 - 2050”, espacio de diálogo y construcción colectiva entre los diferentes actores y sectores claves para la elaboración de insumos, análisis y discusión, que contribuyan a alcanzar la seguridad hídrica al 2050 (Escenarios hídricos 2030, 2019a). Para ello, se diseñó una metodología colectiva con cinco etapas al 2019, bajo un enfoque de innovación social, una colaboración radical y sinérgica, sobre la base de un objetivo común, que busca el uso racional, compartido y sustentable del agua (Escenarios hídricos 2030, 2018).

En la primera etapa, se construyó gobernanza en diferentes niveles de gobierno y con la participación de actores sociales públicos, privados, no gubernamentales, entre otros (Escenarios hídricos 2030, 2018). En la segunda etapa, se elaboraron indicadores sobre la situación actual y tendencia en el tiempo del recurso hídrico en 
Chile (Escenarios Hídricos 2030, 2018). Para la tercera etapa, se formularon escenarios hídricos al 2030 y 2050, a partir, de los cual, se construyeron hojas de ruta de la cuarta etapa, que priorizan las inversiones e intervenciones para fortalecer el desarrollo y la seguridad hídrica (Escenarios hídricos 2030, 2019b). La quinta y última etapa es la difusión y comunicación de los resultados (Escenarios hídricos 2030, 2018).

Los escenarios hídricos, se constituyen en un caso de implementación de la función de gobernanza del agua asociada a "Gestión de desastres (riesgo)", articulada a otras funciones como la "Política y estrategia" y la "Coordinación", entre otros.

LA-Wet Net. La Red Latinoamericana de Desarrollo de Capacidades para la Gestión Integrada del Agua, LA-WETnet, conformada en Perú, en 2002, se creó como respuesta a los compromisos asumidos por los estados miembros de las Naciones Unidas, en el 2000, que condujeron a las Metas del Milenio (CAP-Net UNDP, 2019).

LA-WETnet es, hoy, una red regional, autónoma y sin fines de lucro, que ofrece servicios de desarrollo de capacidades de alta calidad y costos accesibles, sobre la gestión integrada y sostenible del agua y el acceso al agua y el saneamiento, en un contexto de cambio climático (LA-Wet Net, 2019). Para ello, se plantea, como misión, conformar una red de organizaciones y personas dedicadas a la enseñanza, investigación y gestión del agua, con el fin de fortalecer las capacidades de los distintos grupos de interés en América Latina, para una gestión sostenible del agua y una mejora en el acceso al agua y al saneamiento, contribuyendo a la salud, la protección ambiental y a la reducción de la pobreza en la región (LA-Wet Net, 2019).

Desde el inicio de actividades en 2003, la Red ha realizado un total de 85 actividades de desarrollo de capacidades, la mayor parte, cursos presenciales, con lo cual, se ha tenido una cobertura de 3.132 personas. En 2016, se iniciaron los cursos virtuales, como complemento a los presenciales y en colaboración con el Campus Virtual de Cap-Net PNUD (LA-Wet Net, 2019), red internacional para el desarrollo de capacidades en la gestión integrada de los recursos hídricos, a la cual, está afiliada desde 2002.

LA-WETnet, se constituye en un caso de "Desarrollo de capacidades" en el sector de la GIRH en Latinoamérica, función del marco de gobernanza del agua, que requiere, también, de la "Coordinación" con instituciones y organizaciones del sector, como GWP y UNDP, para el cumplimiento de su misión, como dinamizadora y articuladora del conocimiento de la GIRH, en la región.

Para concluir, el cumplimiento, a nivel global del ODS 6, requiere de nuevos enfoques que integren el APSH y la GIRH, sectores que, tradicionalmente, se han visto como separados dentro de la gestión del agua y, en algunos casos, competidores por el recurso hídrico. Es allí, donde modelos más adecuados de gobernanza del agua son fundamentales para poder entender la manera como operan estos sectores, permitiendo aumentar su integración y potenciar los resultados que se requieren para lograr el ODS 6; no obstante, se debe tener en cuenta que la aplicación de un modelo de gobernanza del agua no genera, de manera per se, la vinculación entre APSH y GIRH, por lo que su mayor virtud está en servir de marco de análisis para una eventual integración.

Al analizar los casos de estudio registrados bajo el modelo de gobernanza del agua de SIWI (2018), se identifica que, si bien cada uno de ellos se enmarca en una función principal de gobernanza, todos requieren de "Coordinación" y "Política y Estrategia", como actividades esenciales y complementarias, para alcanzar los fines esperados. Específicamente, la "Coordinación", como una actividad núcleo en la que confluyen y se alinean los intereses, expectativas y necesidades hídricas de las diferentes partes interesadas y la "Política y Estrategia", como una actividad que permite establecer un marco de acción conjunto para la gestión del agua, de cada parte interesada.

Además, se observa cómo las funciones de "Monitoreo, Evaluación y Aprendizaje" y de "Gestión de desastres" son las que menos se registran en los casos estudiados, lo que refleja la baja cultura de seguimiento y evaluación, pero, sobre todo, una débil incorporación de procesos de aprendizaje en el tema. Igualmente, se reconoce una escasa inclusión del riesgo en la toma de decisiones sobre la gestión del agua superficial que, en escenarios de cambio climático, es fundamental para la seguridad hídrica de la región.

Si bien en América Latina se observan ejemplos de cómo se han integrado de manera positiva los sectores de APSH y GIRH, todavía persisten marcos institucionales sectoriales en la gestión del agua, que dificultan la integración de estos sectores, con miras al cumplimiento del ODS6. Es el caso de aspectos estructurales en la gestión del agua, como la formulación de políticas públicas y normatividad y la coordinación, que se siguen desarrollando por cada sector, de manera independiente.

Finalmente, es necesario que los gobiernos latinoamericanos, sobre todo del nivel central, flexibilicen los actuales marcos institucionales de gestión del agua, permitiendo una participación más activa de las diferentes partes interesadas en las decisiones que se toman sobre el recurso hídrico. Es así, como una formulación de políticas y de normas hídricas nacionales más incluyentes y el establecimiento de espacios de coordinación con una mejor representatividad social, entre otros, pueden ayudar en la integración de los sectores de APSH y GIRH y, por consiguiente, a un cumplimiento más efectivo del ODS6 en la región.

Agradecimientos. El autor expresa su agradecimiento al instituto SIWI, por la orientación metodológica para desarrollar el presente artículo. Conflictos de intereses: Este manuscrito fue preparado y revisado por el autor, quien declara que no existe ningún conflicto de intereses que ponga en riesgo la validez de los resultados presentados. Financiación: Este estudio fue elaborado en parte con recursos económicos aportados por la Universidad del Valle. 


\section{REFERENCIAS}

1. ABRIL, A.; NOE, L.; FIILIPINNI, M. 2014. Manejo de enmiendas para restaurar la materia orgánica del suelo en oasis de regadío de Mendoza, Argentina. Revista de Investigaciones Agropecuarias RIA INTA (Argentina). 40:83-91. Disponible desde Internet en: https://www.redalyc.org/articulo.oa?id=86430499014 (con acceso el 08/01/2020).

2. ALTA COMISIONADA DE LAS NACIONES UNIDAS PARA LOS DERECHOS HUMANOS, ACNUDH. 2019. Informe sobre la situación de los derechos humanos en el República Bolivariana de Venezuela (Suiza). Disponible desde Internet en:

https: / / www.ohchr.org/SP/NewsEvents/Pages / DisplayNews.aspx?NewsID $=24788 \&$ LangID $=S$ (con acceso el 04/02/2020).

3. ARGOTE, K. 2019. Blog CGIAR. Disponible desde Internet en:

https: / / blog.ciat.cgiar.org/es/peru-avanza-en-laimplementacion-de-la-ley-de-mecanismos-de-retribucionpor-servicios-ecosistemicos/ (con acceso el 04/12/2019).

4. ARROYO, V., BALLESTERO, M., MEJÍA, A. 2015. Inseguridad Económica del Agua en Latinoamérica: de la abundancia a la inseguridad. CAF (Venezuela). Disponible desde Internet en:

https://scioteca.caf.com/handle/123456789/787 (con acceso el 15/12/2019).

5. BUTTERWORTH, J.; WARNER, J.; MORIARTY, P.; SMITS, S.A. 2010. Finding practical approaches to Integrated Water Resources Management. Water Alternatives 3(1):68-81.

6. CAP-NET UNDP. 2019. La red. Disponible desde Internet en: http://www.cap-net.org/es/partners/la-wetnet-2/ (con acceso el 15/11/2019).

7. CAP-NET UNDP; GLOBAL WATER PARTNERSHIP-GWP. 2004. Planes de gestión integrada del recurso hídrico, Manual de capacitación y guía operacional. Disponible desde Internet en:

https://www.gwp.org/globalassets/global/gwp-cam_files/ manual-planes-girh.pdf (con acceso el 2/01/2020).

8. CHAFLA, P.; CERON, P. 2016. Pago por servicios ambientales en el sector del agua: El Fondo para la Protección de Agua. Tecnología y ciencias del agua. 7(6):25-40. Disponible desde Internet en:

https:// www.redalyc.org/articulo.oa?id=353549830002 (con acceso el 3/02/2020).
9. CONGRESO DE LA REPÚBLICA DEL PERÚ. 2013. Ley de Modernización de los Servicios Públicos. Ley 30045. Lima, Perú: Congreso de la Republica del Perú. Disponible desde Internet en:

https://busquedas.elperuano.pe/normaslegales/apruebanel-reglamento-de-la-ley-n-30045-ley-de-modernizacdecreto-supremo-n-013-2016-vivienda-1408435-1/ (con acceso el 9/09/2019).

10. CONSEJO DEPARTAMENTAL DE POLÍTICA AMBIENTAL Y DE GESTIÓN INTEGRAL DEL RECURSO HÍDRICO, CODEPARH. 2019. Informe de gestión 2016 - 2018. Gobernación del Valle del Cauca (Colombia). Disponible desde Internet en:

http://www.valledelcauca.gov.co (con acceso el 12/10/2019).

11. COOPERACIÓN TÉCNICA BELGA, CTB ENABEL. 2019. Sincronías. La experiencia boliviana de la política pública de cuencas. Rotembol Impresiones (Bolivia). Disponible desde Internet en:

https://www.enabel.be/sites/default/files/sincronias_pnc. pdf (con acceso el 11/11/2019).

12. DENMARK'S DEVELOPMENT COOPERATION, DANIDA. 2007. Apoyo al Desarrollo Sostenible, la Gestión Ambiental y el Manejo de Recursos Naturales. Apoyo al Plan Nacional de Cuencas. (Bolivia). Disponible desde Internet en:

http://bolivia.um.dk/es/ /media/Bolivia/Documents/ Content $\% 20$ Spanish/Subcomponent1d.doc (con acceso el 11/01/2020).

13. DEPARTAMENTO GENERAL DE IRRIGACIÓN, DGI. 2019a. Aquabook. Disponible desde Internet en: http:/ /aquabook.agua.gob.ar (con acceso el 17/01/2020).

14. DEPARTAMENTO GENERAL DE IRRIGACIÓN, DGI. 2019b. Disponible desde Internet en: http://www.irrigacion.gov.ar/dgi/sites/default/ files/2018-11/Resolucion_400-2003_del_HTA.pdf (con acceso el 18/01/2020).

15. ESCENARIOS HÍDRICOS 2030. 2018. Radiografía del agua: Brecha y Riesgo hídrico en Chile. Santiago de Chile: Fundación Chile. Disponible desde Internet en: https:/ / fch.cl/wp-content/uploads/2019/12/resumenradiografia-del-agua-1.pdf (con acceso el 01/02/2020).

16. ESCENARIOS HÍDRICOS 2030. 2019a. Transición hídrica: El futuro del agua en Chile. Resumen Estratégico. Santiago de Chile: Fundación Chile. Disponible desde Internet en: https:/ / fch.cl/wp-content/uploads/2019/12/transicionhidrica-resumen.pdf (con acceso el 01/02/2020). 
17. ESCENARIOS HÍDRICOS 2030. 2019b. Escenarios Hídricos. Disponible desde Internet en:

https:// www.escenarioshidricos.cl/quehacemos/\#hojaderuta (con acceso el 01/02/2020).

18. FLAMMINI, A.; PURI, M.; PLUSCHKE, L.; DUBOIS, O. 2014. Walking the Nexus Talk: Assessing the WaterEnergy-Food Nexus in the Context of the Sustainable Energy for All Initiative. FAO (Italia). Disponible desde Internet en:

http://www.fao.org/3/a-i3959e.pdf (con acceso el 18/02/2020).

19. FORESI, C.H. 2017. El uso de aguas residuales tratadas en Mendoza - Argentina. En: Hettiarachchi, H; Ardakanian, R. Uso seguro de las aguas residuales en la agricultura: Ejemplos de buenas prácticas. UNU-FLORES (Alemania). p.261-267.

20. GIL-BEROES, M. 2020. Entrevista sobre el monitoreo y evaluación del agua en Venezuela. (G. Fernández-Vargas, Entrevistador).

21. GIORDANO, M.; SHAH, T. 2014. From IWRM back to integrated water resources management. International Journal of Water Resources Development. 30(3):364-376. https://doi.org/10.1080/07900627.2013.851521

22. GLOBAL WATER PARTNERSHIP, GWP. 2019. Disponible desde Internet en: https://www.gwp.org/en/learn/iwrm-toolbox/About_ IWRM_ToolBox/(con acceso el 25/01/2020).

23. GLOBAL WATER PARTNERSHIP, GWP. 2000. Gestión Integrada de Recursos Hídricos. TAC Background Papers 4. (Chile). Disponible desde Internet en: https://www.gwp.org/globalassets/global/toolbox/ publications/background-papers/04-integrated-waterresources-management-2000-spanish.pdf (con acceso el 18/02/2020).

24. GOBERNACIÓN DEL VALLE DEL CAUCA. 2019. Plan de Desarrollo Departamental 2026-2019 “El Valle está en vos”. Gobernación del Valle (Colombia). Disponible desde Internet en: https://www.valledelcauca.gov.co/loader.php?1Servic io $=$ Tools2\&lTipo $=$ viewpdf\&id $=40516($ con acceso el 01/02/2020).

25. GOLDMAN-BENNER, R.L.; BENITEZ, S.; BAUCHER, T; CALVACHE, A.; DAILY, G.; KAREIVA, P.; KROGER, T.; RAMOS, A. 2012. Water funds and payments for ecosystem services: practice learns from theory and theory can learn from practice. Oryx. 46(1):55-63. https://doi.org/10.1017/S0030605311001050
26. GRUPO ORINOCO. 2018. Hoja de ruta para recuperar los servicios de Agua Potable y Saneamiento en Venezuela. Grupo Orinoco (Venezuela). Disponible desde Internet en:

https://orinocodotblog.files.wordpress.com/2018/10/ ruta_del_agua_181010.pdf (con acceso el 15/01/2020).

27. HAVEKES, H.; HOFSTRA, M.; KERK, A.; TEEUWEN, B. 2013. Building blocks for good water governance. WGC (The Netherlands). Disponible desde Internet en: https://www.uvw.nl/wp-content/files/Building $\% 20$ blocks $\% 20$ for $\% 20$ water $\% 20$ good $\% 20$ governance $\% 20$ 2013.pdf (con acceso el 25/01/2020).

28. JOSLINA, A.J.; JEPSON, W.E. 2018. Territory and authority of water fund payments for ecosystem services in Ecuador's Andes. Geoforum. 91:10-20. https://doi.org/10.1016/j.geoforum.2018.02.016

29. LEGISLATURA DE MENDOZA. 2019. EPAS Ente provincial de Agua y saneamiento. Disponible desde Internet en: http://www.epas.mendoza.gov.ar/images/documentos/ usuarios/Ley_Prov._6044.pdf (con acceso el 18/01/2020).

30. MINISTERIO DE MEDIO AMBIENTE Y AGUA. 2013. Programa Plurianual de Gestión Integrada de Recursos Hídricos y Manejo Integral de Cuencas 2013 y 2017. Viceministerio de Recursos Hídricos y Riego (Bolivia). Disponible desde Internet en: https://www.researchgate.net/publication/281244646 (con acceso el 19/01/2020).

31. MINISTERIO DE MEDIO AMBIENTE Y AGUA. 2017. Programa Plurianual de Gestión Integrada de Recursos Hídricos y Manejo Integral de Cuencas 2017 y 2020. Viceministerio de Recursos Hídricos y Riego (Bolivia). Disponible desde Internet en:

https://datos.siarh.gob.bo/biblioteca/250 (con acceso el $19 / 01 / 2020)$.

32. MINISTERIO DEL AGUA. 2007. Marco Conceptual y Estratégico del Plan Nacional de Cuencas. Viceministerio de Cuencas y Recursos Hídricos (Bolivia). Disponible desde Internet en: https://www.bivica.org/file/view/id/669 (con acceso el $19 / 01 / 2020)$.

33. OPPLIGER, A.; HÖHL, J.; FRAGKOU, M. 2019. Escasez de agua: develando sus orígenes en la cuenca del Rio Bueno, Chile. Revista de Geografía Norte Grande (Chile). 73:9-27. https://doi.org/10.4067/S0718-34022019000200009

34. ORGANIZACIÓN PARA LA COOPERACIÓN Y EL DESARROLLO ECONÓMICOS, OCDE. 2012. Gobernabilidad del Agua en América Latina y el Caribe. 
Un enfoque multinivel. OCDE (Francia). Disponible desde Internet en:

https://read.oecd-ilibrary.org/governance/gobernabilidaddel-agua-en-america-latina-y-el-caribe_9789264079779es\#page1 (con acceso el 21/01/2020).

35. PINTO, M. 2012. Estrategias de adaptación al cambio climático desde la Política y Legislación de Aguas en Argentina. Actas de Derecho de Aguas (Chile). 2012:143-186. Disponible desde Internet en:

https://www.researchgate.net/publication/291357140_ Estrategias_de_adaptacion_al_cambio_climatico_desde_ la_politica_y_legislacion_de_aguas_en_Argentina (con acceso el 21/03/2020).

36. QUINTERO, M.; PAREJA, P. 2015. Estado de Avance y Cuellos de Botella de los Mecanismos de Retribución por Servicios en Perú. Centro Internacional de Agricultura Tropical CIAT (Colombia). 40p. Disponible desde Internet en: http://www.bosquesandinos.org/wp-content/ uploads/2016/01/cuellos_botella_2015_digital.pdf (con acceso el 10/09/2019).

37. RED LATINOAMERICANA DE DESARROLLO DE CAPACIDADES PARA LA GESTIÓN INTEGRADA DEL AGUA, LA-Wet NET. 2019. Disponible desde Internet en:

https://lawetnet.org/acerca-de-la-wetnet/ (con acceso el 18/01/2020).

38. RED VENEZOLANA DE ORGANIZACIONES DE LA SOCIEDAD CIVIL, SINERGIA. 2019. Los objetivos de desarrollo sostenible en Venezuela. Reporte desde un país en riesgo. SINERGIA (Venezuela). 155p. Disponible desde Internet en:

http://sinergia.org.ve/2019/07/22/los-objetivos-dedesarrollo-sostenible-en-venezuela-reporte-de-un-paisen-riesgo/ (con acceso el 18/02/2020).

39. RED VENEZOLANA DE ORGANIZACIONES DE LA SOCIEDAD CIVIL, SINERGIA. 2020. Agenda Venezuela 2030. SINERGIA (Venezuela). Disponible desde Internet en:

https://www.agendavenezuela2030.org/ (con acceso el 18/02/2020).

40. RESTREPO, I. 2004. Tendencias mundiales en la gestión de recursos hídricos: desafíos para la ingeniería del agua. Ingenieria y competitividad. 6(1):63-71. https://doi.org/10.25100/iyc.v6i1.2289

41. ROGERS, P.; HALL, A.W. 2003. Effective Water Governance. Tec Background Papers No 7. GWP (Suecia). Disponible desde Internet en:

https://www.gwp.org/globalassets/global/toolbox/ publications/background-papers/07-effective-watergovernance-2003-english.pdf (con acceso el 18/11/2019).

42. RUIZ, S.A.; GENTES, I.G. 2008. Retos y perspectivas de la gobernanza del agua y gestión integral de recursos hídricos en Bolivia. European Review of Latin American and Caribbean Studies 85:41-59.

https://doi.org/10.18352/erlacs.9618

43. SANITATION AND WATER FOR ALL, SWA. 2020. SWA Framework. Disponible desde Internet en: https://www.sanitationandwaterforall.org/about/ourwork/priority-areas (con acceso el 18/02/2020).

44. SHAH, T. 2016. Aumentando la seguridad hídrica: la clave para la implementación de los Objetivos de Desarrollo Sostenible. GWP (Suecia). Disponible desde Internet en: https://www.gwp.org/globalassets/global/toolbox/ publications/background-papers/tec22_espanol.pdf (con acceso el 18/11/2019).

45. STOCKHOLM INTERNATIONAL WATER INSTITUTE, SIWI. 2018. Unpacking Governance. Presentación reunión de grupo. SIWI (Suecia).

46. UNITED NATIONS, UN. 2015. Transformar nuestro mundo: la Agenda 2030 para el Desarrollo Sostenible. Asamblea General el 25 de septiembre de 2015. UN (Estados Unidos). 40p. Disponible desde Internet en: https://unctad.org/meetings/es/SessionalDocuments/ ares70d1_es.pdf (con acceso el 12/01/2020).

47. UNITED NATIONS DEVELOPMENT PROGRAMME, UNDP; STOCKHOLM INTERNATIONAL WATER INSTITUTE, SIWI; WATER INTEGRITY NETWORK, WIN. 2013. Users Guide on Assessing Water Governance. UNDP (Noruega). 115p. Disponible desde Internet en: https://www.watergovernance.org/resources/usersguide-on-assessing-water-governance/ con acceso el $13 / 01 / 2020)$.

48. UNITED NATIONS EDUCATIONAL, SCIENTIFIC AND CULTURAL ORGANIZATION, UNESCO. 2006. Water, a Shared Responsibility. World Water Assessment Programme. The United Nations World Water Report 2. UNESCO and Berghahn Books (Francia y USA). Disponible desde Internet en:

https://unesdoc.unesco.org/ark:/48223/pf0000145405 (con acceso el 14/01/2020).

49. UNITED NATIONS INTERNATIONAL CHILDREN'S EMERGENCY FUND, UNICEF. 2016. Strengthening Enabling Environment for Water, Sanitation and Hygiene (WASH): Guidance Note. UNICEF (USA). Disponible desde Internet en: 
https://www.unicef.org/wash/files/WASH_guidance_ note_draft_10_3_hr.pdf (con acceso el 15/01/2020).

50. UNITED NATIONS INTERNATIONAL CHILDREN'S EMERGENCY FUND, UNICEF; UNITED NATIONS DEVELOPMENT PROGRAMME, UNDP; STOCKHOLM INTERNATIONAL WATER INSTITUTE, SIWI. 2016. Enabling environment and water governance. SIWI (Suecia). Disponible desde Internet en:

https://www.watergovernance.org/resources/enablingenvironment-and-water-governance-a-conceptualframework/ (con acceso el 15/01/2020).

51. VALENCIA, C.E.; HERRERA, P.; TIRIBOCCHI, A. 2019. Garantizar la disponibilidad de agua, su gestión sostenible y el saneamiento para todos: implementación de políticas públicas en América Latina y el Caribe. UNESCO y CODIA (España). 240p. Disponible desde Internet en: https://unesdoc.unesco.org/ark:/48223/pf0000370289 (con acceso el 14/01/2020).
52. WETLANDS INTERNATIONAL. 2017. WASH and Water Security: Integration and the role of civil society. Wetlands International (The Netherlands). 50p. Disponible desde Internet en:

https://www.wetlands.org/publications/wash-watersecurity-integration-role-civil-society/ (con acceso el 10/01/2020).

53. ZAAG, P. 2005. Integrated Water Resources Management: Relevant concept or irrelevant buzzword? A capacity building and research agenda for Southern Africa. Physics and Chemistry of the Earth Parts A/B/C 30(11-16):86787.

https://doi.org/10.1016/j.pce.2005.08.032 